\title{
DNA Topoisomerase II Is Not Detectable on Lampbrush Chromosomes but Enriched in the Amplified Nucleoli of Xenopus Oocytes
}

\author{
DAGMAR Fischer, ${ }^{1}$ RoBert Hock, AND UlRICH Scheer \\ Department of Cell and Developmental Biology, Theodor-Boveri-Institute (Biocenter), \\ University of Würzburg, Am Hubland, D-97074 Würzburg, Germany
}

In somatic cells DNA topoisomerase II (topo II) is thought to be involved in the domain organization of the genome by anchoring the basis of chromatin loops to a chromosomal scaffold. Lampbrush chromosomes of amphibian oocytes directly display this radial loop organization in cytological preparations. In order to find out whether topo II may play a role in the organization of these meiotic chromosomes, we performed immunofluorescence studies using antibodies against Xenopus topo II. Our results indicate that topo II is apparently absent from lampbrush chromosomes and is hence unlikely to act as a "fastener" of the numerous lateral chromosomal loops. Topo II was, however, enriched in the amplified nucleoli of Xenopus oocytes. (c) 1993 Academic Press, Ine.

\section{INTRODUCTION}

The chromatin fiber of eukaryotic cells is folded into a series of loops or domains which are stabilized by a structural framework called nuclear matrix or chromosomal scaffold. The loops are thought to be anchored at their base to the matrix or scaffold via specific attachment sites, the so-called matrix or scaffold attachment regions (MARs or SARs). Loop length is variable, ranging from 5 to $200 \mathrm{kbp}$ of DNA [for reviews see 1, 2]. Unfortunately, the chromatin loop structures cannot be directly visualized in interphase nuclei with cytological methods because the chromatin strands are highly entangled.

The situation is different for amphibian lampbrush chromosomes which can be readily examined under almost native conditions by light microscopy [3]. The structural organization of these meiotic chromosomes exhibits a striking similarity to the loop model described above. Each lampbrush chromosome consists of a chromosomal axis from which numerous loops radiate laterally. The axis represents a linear array of chromo-

\footnotetext{
${ }^{1}$ To whom correspondence and reprint requests should be addressed at Zoologie I, Biozentrum Am Hubland, D-97074 Würzburg, Germany. Fax: 00499318884252
}

meres containing highly condensed chromatin, while the lateral loops consist of decondensed, transcriptionally active chromatin covered with numerous nascent transcripts. Because lampbrush chromosome architecture resembles so closely the proposed organization of somatic interphase chromatin and mitotic chromosomes, it is tempting to speculate that the same molecular components might be involved in the maintenance of chromatin loops.

One of the best characterized components of the chromosomal scaffold is DNA topoisomerase II (topo II) [47]. Besides its enzymatic activity that creates transient double-strand breaks in duplex DNA and passes DNA strands through one another, the protein has been shown to interact with the MAR and SAR elements [8]. Thus, topo II is thought to have catalytic as well as structural functions. Topo II is not only associated with the scaffold of mitotic chromosomes but also the cores and centromeric regions of spermatocyte-derived meiotic chromosomes [9]. Recently Luke and Bogenhagen identified by biochemical means topo II in the germinal vesicles of Xenopus oocytes [10]. These findings prompted us to study the localization of topo II in Xenopus germinal vesicles in order to find out whether it is associated with lampbrush chromosomes and hence could play a role in maintaining the higher-order structure of these particular chromosomes.

\section{MATERIAL AND METHODS}

Animals. Female Xenopus laevis were purchased from the South African Snake Farm (Fish Hoek, Cape Province, South Africa).

Antibodies. The rabbit-derived antiserum against Xenopus topo II has been described in detail [10]. Antiserum and preimmune serum were generously provided by Dr. D. Bogenhagen (Stony Brook, New York).

Immunofluorescence microscopy. Xenopus A6 cells were grown on coverslips as described elsewhere [11], rinsed with PBS, fixed with methanol $\left(10 \mathrm{~min},-20^{\circ} \mathrm{C}\right)$ followed by acetone $\left(5 \mathrm{~min},-20^{\circ} \mathrm{C}\right)$ and finally air-dried.

Cryostat sections ( $5 \mu \mathrm{m}$ ) of shock-frozen Xenopus ovary were airdried, fixed in 1\% formaldehyde in PBS (15 min), and rinsed with PBS.

Xenopus oocytes were staged according to Dumont [12]. Lampbrush chromosomes were prepared following the protocol described by Callan et al. [13]. After fixation in $70 \%$ ethanol, slides were transferred into PBS. 
Cells, cryostat sections, or lampbrush chromosome preparations were incubated with antiserum against topo II or corresponding preimmune serum (1:200 diluted in PBS) for $30 \mathrm{~min}$ at room temperature. After three washes in PBS, secondary antibodies were added (FITC-conjugated goat anti-rabbit immunoglobulins; Dianova, Hamburg, Germany; diluted 1:100 in PBS). After an incubation time of 30 $\mathrm{min}$ at room temperature slides or coverslips were again washed in PBS and finally mounted in "antifade" [14] supplemented with the DNA-staining fluorescent dye Hoechst 33258 (final concentration, 5 $\mu \mathrm{g} / \mathrm{ml})$.

Photographs were taken with a Zeiss Axiophot (Carl Zeiss, Oberkochen, Germany) equipped with epifluorescence optics and the appropriate filter sets.

Gel electrophoresis and immunoblotting. Proteins were resolved by SDS-PAGE [15] using 6\% acrylamide in the separating gel. For immunoblots, proteins were transferred to nitrocellulose [16]. The membrane was blocked with $5 \%$ nonfat dry milk in TBS $(10 \mathrm{mM}$ Tris/ $\mathrm{HCl}, \mathrm{pH} 7.4,150 \mathrm{mM} \mathrm{NaCl}$ ) and incubated with topo II-specific antiserum (diluted 1:20,000 in blocking buffer) for $1 \mathrm{~h}$. After three washes in TBS, secondary antibodies (alkaline phosphatase-conjugated anti-rabbit IgG; Promega, Heidelberg, Germany) were added at $1: 10,000$ dilution in blocking buffer. After three washes in TBS, detection of bound antibodies was performed using nitro blue tetrazolium chloride and 5-bromo-4-chloro-3-indolyl phosphate $p$-toluidine salt (Biomol, Hamburg, Germany) as chromogenic substrates.

Egg extract and quantitation of protein. Xenopus egg extract was prepared according to Newport [17]. Protein was quantified as described by Bradford [18].

\section{RESULTS}

\section{Immunoblotting Analyses}

The topo II-specific antiserum has been reported to react with an $180-\mathrm{kDa}$ oocyte protein in immunoblotting analysis [10]. Since at least two different isoforms of topo II (170 and $180 \mathrm{kDa}$ ) have been identified by molecular cloning [19], drug inhibition studies [20], or immunological approaches involving monoclonal antibodies [21], it was of interest to find out whether the polyclonal antiserum directed against the $180-\mathrm{kDa}$ form of oocyte topoisomerase II also reacted with the 170$\mathrm{kDa}$ form or other variants of the enzyme. For this purpose proteins of Xenopus germinal vesicles, egg extract, or cultured epithelial cells (harvested during logarithmic phase of growth) were separated by gel electrophoresis, transferred to nitrocellulose, and probed with the topo II-specific antiserum (Fig. 1). As already observed by Luke and Bogenhagen [10], the major immunoreactive protein present in Xenopus eggs had a slightly reduced mobility in SDS-PAGE as compared to oocyte topo II. In contrast, the immunoreactive protein of Xenopus A6 cells migrated faster (with an apparent molecular mass of $170 \mathrm{kDa}$ ) than the oocyte topo II (Fig. 1, lanes 1 and 3). Proteins visible in the lower molecular weight range were stained nonspecifically as judged from control blots using the corresponding preimmune serum (not shown). We conclude that the antiserum used in this study recognizes both the 170- and the 180$\mathrm{kDa}$ form of Xenopus topo II.

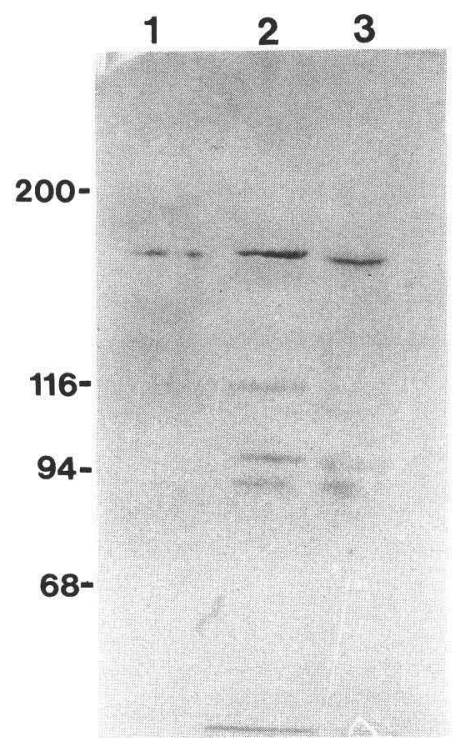

FIG. 1. Immunoblots of isolated oocyte nuclei, eggs, and somatic Xenopus cells with the topo II-specific antiserum. Proteins of $20 \mathrm{Xen-}$ opus oocyte nuclei (lane 1), egg extract (50 $\mu$ g protein) (lane 2), and 2 $\times 10^{6} \mathrm{~A} 6$ cells (lane 3 ) were separated by SDS-PAGE, transferred to nitrocellulose, and probed with the anti-topo II antiserum. In isolated oocyte nuclei and total egg extract, the antibodies bind to a single polypeptide of 180 - or $182-\mathrm{kDa}$, respectively, whereas in $\mathrm{A} 6$ cells the immunoreactive protein has an apparent molecular mass of $170 \mathrm{kDa}$. Molecular masses of the reference proteins are indicated in $\mathrm{kDa}$.

\section{Localization of Topo II in Cultured Xenopus A6 Cells}

Since the antiserum against Xenopus topo II had so far only been used for immunoprecipitation and Western blot analyses [10], we first had to find out whether these antibodies could also be used for immunofluorescence experiments. Incubation of methanol/acetonefixed Xenopus A6 cells with the antiserum against topo II resulted in a very complex immunofluorescence pattern (Fig. 2). As expected, mitotic chromosomes were intensely labeled (Fig. 2a, arrow). In agreement with earlier studies [6, 22], interphase nuclei were variably stained with the anti-topo II antibodies. While some nuclei were completely negative, others showed a finely punctate fluorescence throughout the nucleoplasm. In addition, the antibodies stained the nucleoli intensely. As judged from the immunofluorescence signal, the relative proportion of nucleolar and extranucleolar topo II appeared to be quite variable in different cells, probably depending on their specific growth stage. Preimmune serum caused only a general fluorescence background reaction. Since our results obtained with Xenopus A6 cells were consistent with the distribution of topo II described in other cells and with other topo II-specific antibodies [6, 21-23], we extended the immunofluorescence approach to study the subcellular localization of topo II in Xenopus oocytes. 

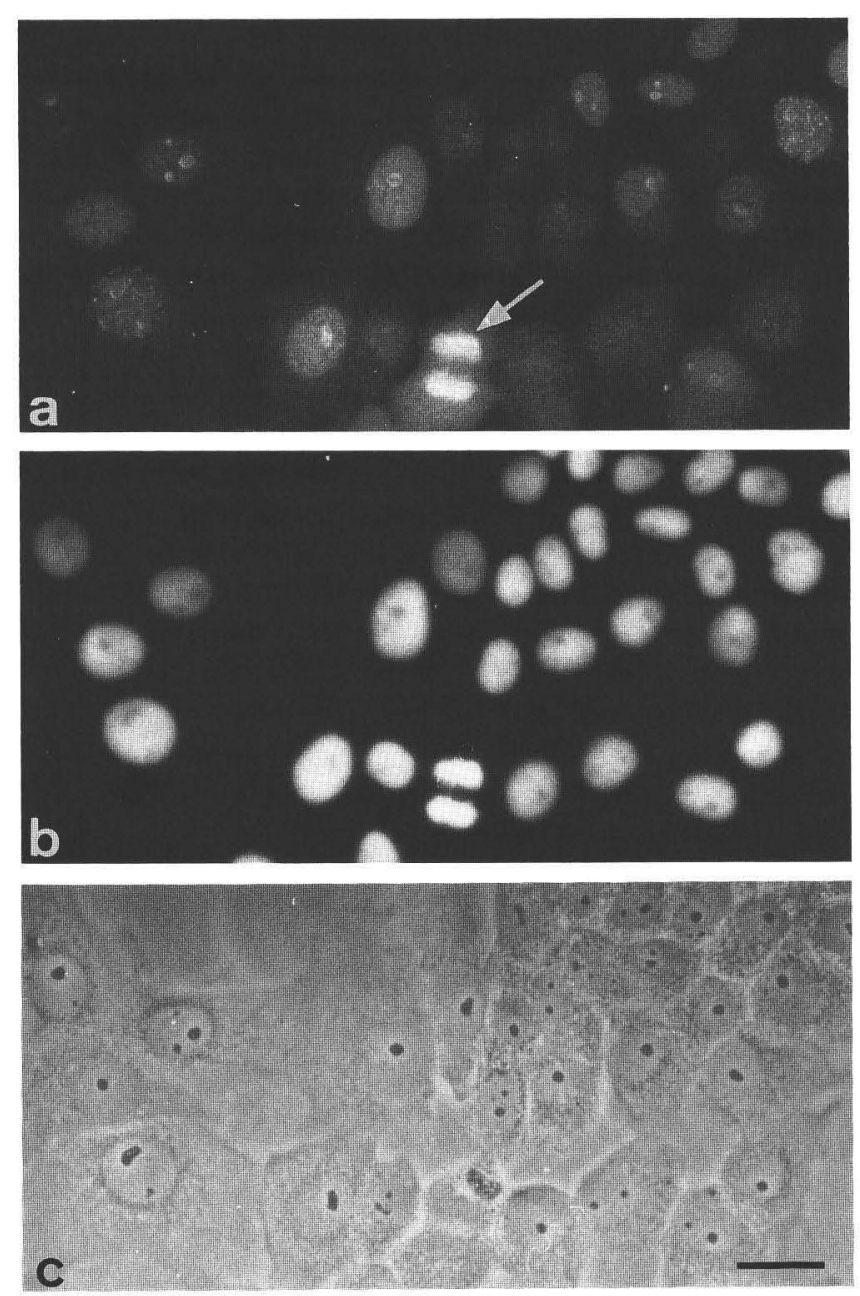

FIG. 2. Immunofluorescence localization of topo II on Xenopus A6 cells. Cells were grown on coverslips and stained with antiserum against topo II. The resulting fluorescence pattern (a) is heterogeneous: Some nuclei are completely negative, some reveal an uniform punctate staining and some a nucleoplasmic plus strong nucleolar staining. Mitotic chromosomes are intensely labeled (arrow in a). The corresponding Hoechst staining (b) and phase-contrast images (c) are shown. Bar: $20 \mu \mathrm{m}$.

\section{Localization of Topo II in Nuclear Structures of Xenopus Oocytes}

As had been shown by Luke and Bogenhagen [10], nuclei of stage V-VI oocytes contain larger amounts of topo II compared to earlier oocyte stages. Therefore, we first examined lampbrush chromosomes prepared from large oocytes. In no case we could detect any significant labeling of the lampbrush chromosomes (Fig. 3a). A faint fluorescence of their axes was also seen with the corresponding preimmune serum and therefore considered to be nonspecific (Fig. 3b). In contrast, the internal component of the amplified nucleoli reacted strongly with the topo II-specific antiserum (Fig. 3a). Lampbrush chromosomes isolated from growing oocytes (stage III-IV) were also negative with the topo II antiserum (Fig. 3c). In these specimens, however, the fluorescence intensity of the amplified nucleoli was much weaker as compared to those prepared from full-grown oocytes (Fig. 3c).

Since it is conceivable that topo II might be extracted during the chromosome isolation procedure (see Discussion), frozen sections of Xenopus ovary were fixed with formaldehyde and processed for immunofluorescence microscopy. The staining pattern was essentially identical to that observed with the spread chromosome preparations. The numerous amplified nucleoli of fullgrown oocytes fluoresced whereas the lampbrush chromosome portions included in the section were negative (data not shown).

\section{DISCUSSION}

The antiserum against the $180-\mathrm{kDa}$ topo II used in this study had already been characterized in detail [10]. It neutralized decatenation activity in egg extracts and reacted in immunoblots with an oocyte protein of approximately $180 \mathrm{kDa}$. The content of topo II increased throughout oogenesis from $0.006 \mathrm{ng}$ in a stage I oocyte to $1.44 \mathrm{ng}$ in a stage VI oocyte with a maximum amount of $3 \mathrm{ng} / \mathrm{egg}$. Luke and Bogenhagen [10] observed that the major band of egg topo II had a slightly reduced mobility in SDS-PAGE compared to the oocyte enzyme. Our immunoblotting data confirm this result and, furthermore, reveal that the antiserum raised against the $180-\mathrm{kDa}$ isozyme of topo II is also crossreacting with a $170-\mathrm{kDa}$ form present in Xenopus A6 cells.

The antiserum against Xenopus $180-\mathrm{kDa}$ topo II is suitable for immunofluorescence studies. In Xenopus A6 cells, mitotic chromosomes and some of the interphase nuclei and nucleoli are stained. Our finding that a subpopulation of the A6 cells did not react with the antibody is in agreement with the results of Earnshaw and colleagues $[6,22]$ who reported that the topo II content of nuclei varies during the cell cycle. They observed that in G1 phase some of the nuclei were negative for topo II. A nucleolar localization of topo II in HeLa cells was also reported by Negri et al. [21]. Using monoclonal antibodies these authors provided evidence for the existence of two immunologically distinguishable isoforms of topo II with different nuclear locations: a $180-\mathrm{kDa}$ form associated with the nucleoli and a $170-\mathrm{kDa}$ form present in the nucleoplasm. While the latter form was more abundant during logarithmic phase of growth, the $180-\mathrm{kDa}$ isoform occurred preferentially during the plateau phase of growth. Recently, however, using polyclonal antisera, Petrov and colleagues [23] observed both isoforms in the nucleoplasm and the nucleolus. Anyhow, since the antiserum used in this study recognizes the 170 - and the $180-\mathrm{kDa}$ form of topo II, it is not difficult to explain that we observed both, a nucleolus-associated as 

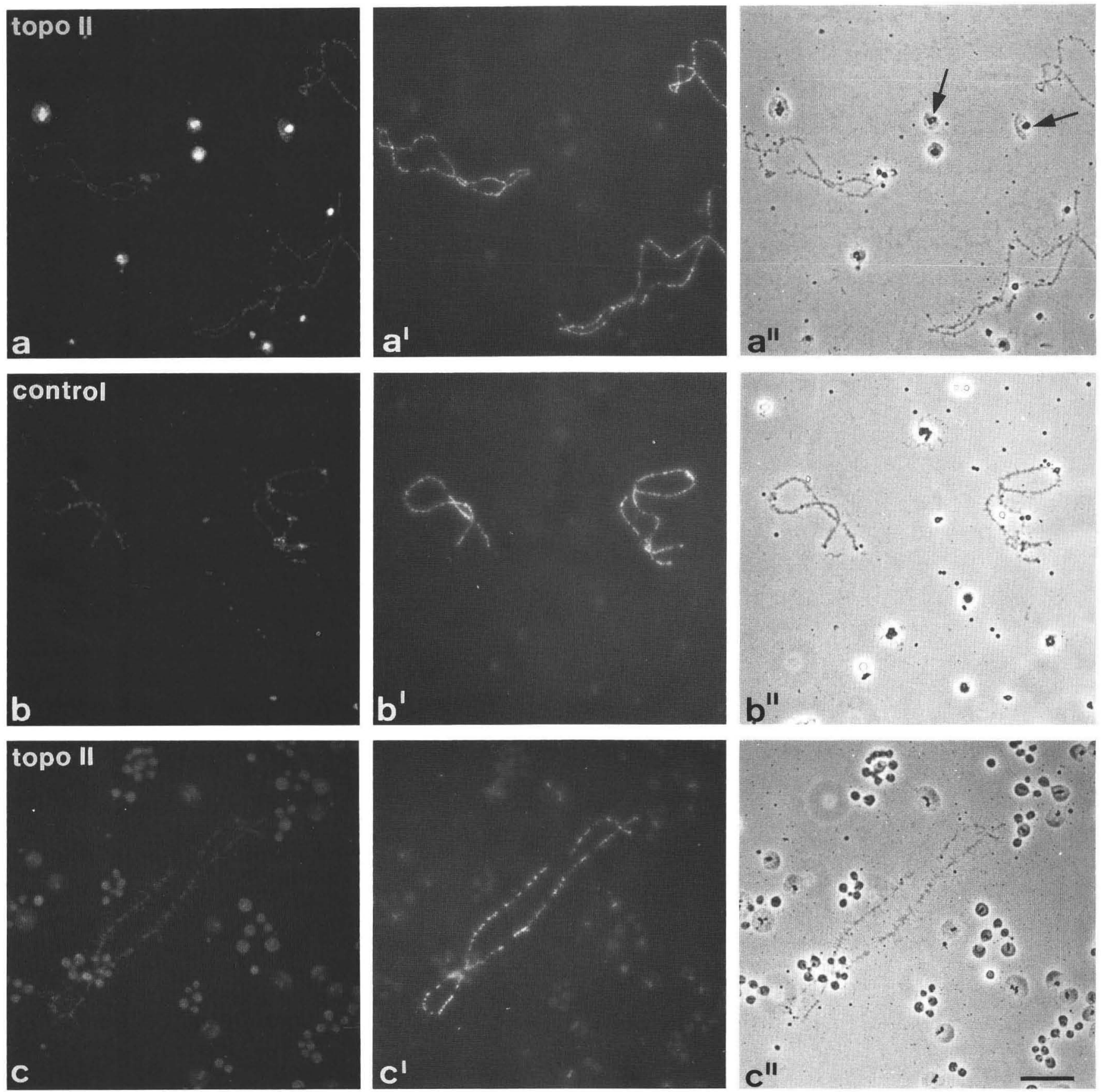

FIG. 3. Immunofluorescence localization of topo II on spread Xenopus lampbrush chromosomes and amplified nucleoli. Spread nuclear contents of stage VI $(a, b)$ or stage IV (c) oocytes were incubated with topo II antiserum $(a, c)$ or corresponding preimmune serum (b). Positive fluorescence is seen exclusively in the amplified nucleoli of mature oocytes, in particular in their inner regions (a), which appear as dense cores in phase contrast (arrows in $\mathrm{a}^{\prime \prime}$ ). The corresponding Hoechst staining is shown in $\mathrm{a}^{\prime}-\mathrm{c}^{\prime}$. Bar: $20 \mu \mathrm{m}$.

well as a nucleoplasmic fluorescence in cultured Xenopus A6 cells.

Using immunofluorescence microscopy we were unable to detect topo II on lampbrush chromosomes prepared from full-grown oocytes known to contain a large store of topo II. However, the topo II-specific antiserum labeled strongly the internal component ("nucleolar core") of the amplified nucleoli which contains the rRNA genes [24]. Antibodies directed against RNA polymerase I produced essentially the same immunofluorescence pattern (data not shown). These findings could be explained by any of the following considerations. 
1. Since previous studies provided evidence for an enrichment of transcriptionally active genes in the nuclear matrix fraction [25, 26], it might be argued that topo II is associated with lampbrush chromosomes only when they are transcribed at maximal rates, i.e., in growing oocytes. We therefore prepared lampbrush chromosomes also from stage IV oocytes. Despite the presence of numerous lateral loops indicative of ongoing transcription, these chromosomes turned out to be negative for topo II as well.

2. Topo II is associated with lampbrush chromosomes but not accessible to the antibodies. Although we cannot formally exclude this possibility, we consider it quite unlikely. Antibodies directed against chromatin constituents such as the core histones [27-29] and DNA [30] label the axis of the lampbrush chromosomes strongly, thus indicating that the specific chromomere organization does not exclude antibody binding. Furthermore, topo II is readily detectable on metaphase chromosomes of somatic cells despite their highly compact chromatin conformation.

3. Topo II is associated with lampbrush chromosomes in vivo but is lost during the isolation procedure. In fact, it has been recently shown that topo II is readily dissociated from mitotic chromosomes assembled in Xenopus egg extract under almost physiological salt conditions (50-100 $\mathrm{mM} \mathrm{NaCl)} \mathrm{[31].} \mathrm{We} \mathrm{therefore} \mathrm{pre-}$ pared lampbrush chromosomes under conditions that should minimize this potential artifact. Immediately after tearing off the nuclear envelope, the chromosomes were centrifuged for only $5 \mathrm{~min}$ and then fixed in $70 \%$ ethanol. Alternatively, fixation was performed with methanol following the procedure for staining tissue culture cells. Since copper ions in a concentration as low as $10^{-8} M$ have been reported to stabilize the chromosomal scaffold [32], we added $1 \mu M \mathrm{CuCl}_{2}$ into the spreading buffer. In no case topo II was detectable on lampbrush chromosomes.

To exclude the possibility of topo II extraction during chromosome isolation and antibody incubation, we also prepared frozen sections of full-grown oocytes and fixed them immediately in formaldehyde prior to incubation with the anti-topo II antibodies. The only detectable fluorescent structures were the amplified nucleoli, whereas the portions of the lampbrush chromosomes included in the sections were negative. Taken together, our data argue that topo II is either absent from the lampbrush chromosomes or occurs at levels far below the topo II value of mitotic chromosomes [for quantitative data see 7]. Thus, our results speak against an involvement of topo II in the structural maintenance of lampbrush chromosomes. Similar results have been recently obtained from studies of mitotic chromosomes assembled in vitro [31]. These authors showed that mitotic chromosomes retained their specific organization after extraction of topo II.
4. One could imagine that lampbrush chromosomes contain a topo II-like protein that is not recognized by the antibody used in our study. Although this cannot be ruled out completely, we consider this unlikely since the antiserum reacts with a 180 - and a $170-\mathrm{kDa}$ form of topo II and neutralizes all decatenation activity in egg extracts. Moreover, Xenopus egg extract depleted of topo II (using the same antiserum as that in the present study) failed to convert chicken erythrocyte nuclei into condensed chromosomes. Upon readdition of purified topo II, condensation activity was restored [33]. These results clearly demonstrate that immunologically distinct proteins with topo II activities are absent in Xenopus eggs.

5. Lampbrush chromosomes lack topo II but may contain other protein(s) involved in anchoring the lateral loops to the chromosomal axis. A precedent for a distinct difference in molecular composition of oocyte lampbrush chromosomes and somatic chromosomes has recently been found. Although chromatin of the lampbrush chromosome axes is highly condensed, histone $\mathrm{H} 1$ seems to be absent [28]. Thus, in spite of the striking similarity of lampbrush chromosome morphology and the loop model of somatic chromatin organization, the molecular mechanisms underlying chromatin folding need not to be identical. Although negative outcomes of immunocytochemical labeling experiments should be cautiously interpreted, we favor the view that topo II is in fact absent from lampbrush chromosomes.

The question remains: What is the function of topo II associated with the amplified nucleoli in full-grown Xenopus oocytes? As has been shown in several experimental systems, topo II is essential for chromosome condensation and segregation during mitosis as well as during meiosis [31, 33-43]. It is one of the characteristics of Xenopus development that during the early, rapid embryonic cleavages there is almost no transcriptional activity. Until the midblastula transition the embryo is therefore dependent on maternally stored transcripts and proteins including topo II [44]. It remains to be elucidated whether topo II bound to the amplified nucleoli represents a storage form of this enzyme for future embryonic development and/or plays a direct role in the structural and functional organization of these nucleoli. The finding that topo II is also found enriched in nucleoli of somatic cells $[21,23]$ supports the view that it is a genuine component of nucleoli in general.

We thank Drs. May Luke and Daniel Bogenhagen for their generous gift of topo II-specific antiserum and Hilde Merkert for photographical work. Our work has been supported by a grant of the Deutsche Forschungsgemeinschaft (Sche 157/9-1).

\section{REFERENCES}

1. Gasser, S. M., and Laemmli, U. K. (1987) Trends Genet. 3, 1622. 
2. Earnshaw, W. C. (1988) BioEssays 9, 147-150.

3. Callan, H. G. (1986) Mol. Biol. Biochem. Biophys. 36, 1-254.

4. Berrios, M., Osheroff, N., and Fisher, P. A. (1985) Proc. Natl. Acad. Sci. USA 82, 4142-4146.

5. Earnshaw, W. C., and Heck, M. M. S. (1985) J. Cell Biol. 100, 1716-1725.

6. Earnshaw, W. C., Halligan, B., Cooke, C. A., Heck, M. M. S., and Liu, L. F. (1985) J. Cell Biol. 100, 1706-1715.

7. Gasser, S. M., Laroche, T., Falquet, J., Boy de la Tour, E., and Laemmli, U. K. (1986) J. Mol. Biol. 188, 613-629.

8. Adachi, Y., Käs, E., and Laemmli, U. K. (1989) EMBO J. 8, 3997-4006.

9. Moens, P. B., and Earnshaw, W. C. (1989) Chromosoma 98, 317-322.

10. Luke, M., and Bogenhagen, D. F. (1989) Dev. Biol. 136, 459468.

11. Smith, J. C., and Tata, J. R. (1991) Methods Cell Biol. 36, 635654.

12. Dumont, J. N. (1972) J. Morphol. 136, 153-180.

13. Callan, H. G., Gall, J. G., and Berg, C. A. (1987) Chromosoma 95, 236-250.

14. Johnson, G. D., and Nogueira Araujo, G. M. (1981) J. Immunol. Methods 43, 349-350.

15. Laemmli, U. K. (1970) Nature 227, 680-685.

16. Kyhse-Andersen, J. (1984) J. Biochem. Biophys. Methods 10, 203-209.

17. Newport, J. (1987) Cell 48, 205-217.

18. Bradford, M. M. (1976) Anal. Biochem. 72, 248-254.

19. Chung, T. D. Y., Drake, F. H., Tan, K. B., Per, S. R., Crooke, S. T., and Mirabelli, C. K. (1989) Proc. Natl. Acad. Sci. USA 86, 9431-9435

20. Holden, J. A., Rolfson, D. H., and Wittwer, C. T. (1990) Biochemistry 29, 2127-2134.

21. Negri, C., Chiesa, R., Cerino, A., Bestagno, M., Sala, C., Zini, N., Maraldi, N. M., and Astaldi Ricotti, G. C. B. (1992) Exp. Cell Res. 200, 452-459.

22. Heck, M. M. S., and Earnshaw, W. C. (1986) J. Cell Biol. 103, $2569-2581$.
23. Petrov, P., Drake, F. H., Loranger, A., Huang, W., and Hancock, R. (1993) Exp. Cell Res. 204, 73-81.

24. Miller, O. L., and Beatty, B. R. (1969) Science 164, 955-957.

25. Cook, P. R., Lang, J., Mayday, A., Lania, L., Fried, M., Chiswell, D. J., and Wyke, J. A. (1982) EMBO J. 1, 447-452.

26. Robinson, S. J., Nelkin, B. D., and Vogelstein, B. (1982) Cell 28, 99-106.

27. Scheer, U., Sommerville, J., and Bustin, M. (1979) J. Cell Sci. 40, 1-20.

28. Hock, R., Moorman, A., Fischer, D., and Scheer, U. (1993) Dev. Biol. 158, 510-522.

29. Sommerville, J., Baird, J., and Turner, B. M. (1993) J. Cell Biol. 120, 277-290.

30. Scheer, U., Messner, K., Hazan, R., Raska, I., Hansmann, P., Falk, H., Spiess, E., and Franke, W. W. (1987) Eur. J. Cell Biol. 43, 358-371.

31. Hirano, T., and Mitchison, T. J. (1993) J. Cell Biol. 120, 601612.

32. Lewis, C. D., and Laemmli, U. K. (1982) Cell 29, 171-181.

33. Adachi, Y., Luke, M., and Laemmli, U. K. (1991) Cell 64, 137148.

34. Holm, C., Goto, T., Wang, J. C., and Botstein, D. (1985) Cell 41, 553-563.

35. Uemura, T., Ohkura, H., Adachi, Y., Morino, K., Shiozaki, K., and Yanagida, M. (1987) Cell 50, 917-925.

36. Holm, C., Stearns, T., and Botstein, D. (1989) Mol. Cell. Biol. 9, 159-168.

37. Wright, S. J., and Schatten, G. (1990) Dev. Biol. 142, 224-232.

38. Charron, M., and Hancock, R. (1990) Biochemistry 29, 95319537.

39. Charron, M., and Hancock, R. (1991) Chromosoma 100, 97-102.

40. Downes, C. S., Mullinger, A. M., and Johnson, R. T. (1991) Proc. Natl. Acad. Sci. USA 88, 8895-8899.

41. Shamu, C. E., and Murray, A. W. (1992) J. Cell Biol. 117, 921 934.

42. Rose, D., Thomas, W., and Holm, C. (1990) Cell 60, 1009-1017.

43. Clarke, D. J., Johnson, R. T., and Downes, C. S. (1993) J. Cell Sci. 105, 563-569.

44. Almouzni, G., and Wolffe, A. P. (1993) Exp. Cell Res. 205, 1-15.

Received April 29, 1993

Revised version received August 27, 1993 